\title{
ONE BUBBLE INFLATION
}

TAKAHIRO TANAKA

Department of Earth and Space Science, Graduate School of Science, Osaka University, Toyonaka 560, Japan

\begin{abstract}
Evaluating the temperature fluctuation in the cosmic microwave background radiation, the viability of the one bubble inflation scenario is studied. It is shown that it is possible to construct a model which explains the creation of an open universe.
\end{abstract}

\section{Introduction}

"Is it possible to say something about the value of cosmological parameters, such as $\Omega$ and $\Lambda$ from the study of the very early universe or the particle cosmology?" One may say that the inflationary universe scenario favors the Einstein deSitter model. That is true. But is it also true that the $\Lambda$-model is prefered to the open model? I think that the answer to this question is "NO". This is the point that I want to explain in my talk.

Why is the inflationary universe scenario thought to be attractive? It is because the inflationary universe scenario can solve many problems about the initial condition of our universe, such as the flatness problem, the homogeneity problem, the entropy problem and so on. They might be categolized into two classes. The flatness problem, the homogeneity problem and many other problems are solved by the accelerated expansion of the length scale. On the other hand, the entropy problem is not directly solved by the accelerated expansion of the universe. It is solved as a result of the release of the vacuum energy after inflation. In other words, the solution of the homogeneity problem means the small excitation in long wavelength modes and that of the entropy problem means the large excitation in short wavelength modes. Inflation explains these two different requirements in a unified picture. That is the attractive point.

Then is it possible to create an open universe in the standard inflationary universe scenario? The answer is "no". As mentioned above, the flatness problem is tightly related to the homogeneity problem. So if we 
want to solve the homogeneity problem, the flatness problem is solved automatically. To see this, let us consider the Friedmann equation in an open universe: $H^{2}(t)=1 / a^{2}(t)+\Omega(t) H^{2}(t)$, where $H:=\dot{a} / a$. This equation can be rewritten into

$$
H^{-1}(t)=\sqrt{1-\Omega(t)} a(t)=p \sqrt{1-\Omega(t)}(a(t) / p),
$$

where $p$ is the comoving wave number. The left hand side, $H^{-1}(t)$ represents the Hubble horizon scale, and $a(t)$ can be understood as the spatial curvature scale. So the first equality shows (a) that in an open universe $\left(1-\Omega_{o}=O(1)\right)$ the curvature scale is comparative to the present horizon scale and (b) that the curvature scale stays outside the horizon scale through the whole history of the universe. Noting that $(a(t) / p)$ is the physical length scale of the perturbation mode specified by the comoving wave number $p$, we find $p \sim 1$ for the perturbation mode corresponding to the present horizon scale. Thus for such a horizon scale mode $a(t) / p \sim a(t) \geq H^{-1}(t)$ holds. That is, the present horizon scale perturbation stays outside the horizon scale. In general, the long wave length mode beyond the horizon scale does not have any effective damping mechnism. So in order that the present horizon scale perturbation mode has a small amplitude at the present epoch, it must have been set so from the beginning. However, the scale of such a mode never becomes smaller than the curvature scale or the horizon scale. This means that the homogeneity problem is not solved by the inflation if we want to construct an open model. As a solution to this difficulty, the one-bubble inflation scenario was proposed.

\section{One-bubble inflation model}

The original idea of the one-bubble inflation scenario was proposed by Gott III(1982). Although there is a more natural model (the two-field model), here we consider the single-field model to explain the basic idea of the one-bubble inflation.

In the single-field model the form of the potential shown in Fig. 1 is assumed. Initially the field is supposed to be trapped in the false vacuum, where the vacuum energy drives the universe to expand exponentially. This exponential expansion solves the homogeneity problem. After sufficiently long inflation, the bubble nucleation occurs through quantum tunneling effect. This process is described by the $O(4)$-symmetric bounce solution. The bounce solution is a solution of the Euclidianised equation of motion that connects the intial and final configurations before and after tunneling. In the lowest WKB order, the state after tunneling is determined by the analytic continuation of this bounce solution. After analytic continuation, the $O(4)$-symmetry becomes the $O(3,1)$-symmetry, which is the symmetry of an open Friedmann-Robertson-Walker universe. So in the lowest order approximation, we have obtained a model that explains the creation of an open and homogeneous universe. 


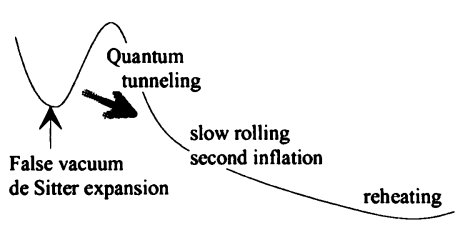

FIG. 1. The schematic picture of the inflaton potential of the singlefield model.

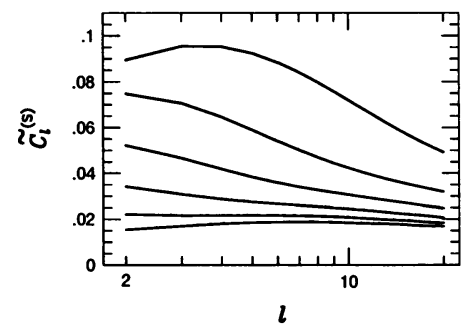

FIG. 2. The normalized scalar CMB anisotropy spectra for various values of $\Omega_{0}$. The lines show, from top to bottom, the cases of $\Omega_{0}=0.1,0.2,0.3,0.4,0.5$ and 0.6 .

However, at this stage the universe is almost empty and the cosmic expansion is dominated by the curvature term in the Friedmann equation. The curvature term decreases slower than any other ordinary matter energy density. Hence, as long as ordinary matter is assumed, the universe stays curvature dominant forever. Therefore the second inflation inside this nucleated bubble is necessary for the matter production.

\section{The spectrum of the cosmological perturbation predicted by the one-bubble inflation model}

In the preceding section, we have seen that the one-bubble inflation scenario give a possible explanation of the creation of an mildly open (i.e., $\Omega \sim 1$ ) and finely homogeneous universe.

To test if this scenario is viable or not, we need to evaluate the predicted spectrum of the cosmological perturbation in this scenario. Here I do not have enough space to explain how to derive the spectrum. So here I just stress that in the context of one-bubble inflation scenario one can predict the spectrum of the cosmological perturbation if the model of the inflaton potential is specified.

In the rest of this section, I explain the essential feature of the predicted spectrum. However, it is hard to discuss the resulting spectrum in general cases. To simplify the analysis here we restrict our consideration to the cases that satisfy the following two assumptions.

1) The mass of the second inflaton field in the false vacuum is sufficiently large compared with $H$.

2) The spacetime inside the nucleated bubble can be approximated by a de Sitter space.

For such models, the multipole moments of the two point correlation function of the temperature fluctuation of the cosmic microwave background radiation, which is defined by

$$
\frac{1}{4 \pi} \sum_{p}(2 \ell+1) C_{\ell} P_{\ell}(\cos \theta)=\left\langle\frac{\delta T}{T}\left(\gamma^{i}\right) \frac{\delta T}{T}\left(\tilde{\gamma}^{i}\right)\right\rangle .
$$


were calculated (Yamamoto et. al. (1996), Sasaki et. al. (1997), Garriga et. al. (1997)) As is known in the standard cosmological perturbation theory, the contribution to the temperature anisotropy can be decomposed into those due to the scalar-type and tensor-type perturbations.

In the cosmological perturbation theory, we decompose the perturbation variables by using the spatial harmonics, which is a set of eigen functions of the Laplacian operator. The square integrability on a time constant hypersurface requires the eigen value $p^{2}$ to be positive. However, the square integrability is not the necessary condition of the normalizablity of the mode with respect to the Klein-Gordon norm. So there can be the supercurvature mode that forms a discrete spectrum with negative $p^{2}$. In the treatment without taking into account the perturbation of the gravitational field, there appeared several so-called supercurvature modes (Yamamoto et. al. (1996)). It is not square integrable but Klein-Gordon normalizable. One of them was the wall fluctuation mode. It is called so because its time dependence is proportional to the time-derivative of the background field. Another is the de Sitter supercurvature mode, which is absent under the assumption 1).

Recently we analyzed the perturbation spectrum fully taking into account the perturbation of the gravitational field (Tanaka et. al. (1997), Sasaki, et. al. (1997), Garriga et. al. (1997)). Then we found that the wall fluctuation mode dessappears from the scalar perturbation spectrum. Instead, the equivalent contribution gives arise at the bottom of the tensor perturbation spectrum. One may think that this is a curious thing because it is believed that the scalar-type and tensor-type perturbations are completely decoupled. However, this belief is not true. In the case of an open universe, the tensor-type harmonics with $p^{2}=0$ and the tensor constructed by differenciating the scalar-type harmonics with $p^{2}=-4$ become degenerate.

As a result, we do not have any supercurvature mode in the scalar-type perturbation. The shape of the continuous spectrum is almost independent of the inflaton potential model except for the curvature scale modes with $p^{2} \lesssim 1$. The contribution of such curvature scale modes to the temperature fluctuation is small. Thus resulting $C_{\ell}$ can be written as $\ell(\ell+1) C_{\ell}^{(S)} \sim$ $\left(3 H^{2} / 5 \dot{\phi}\right)^{2} \tilde{C}_{\ell}^{(S)}$, and $\tilde{C}_{\ell}^{(S)}$ depends only on the value of $\Omega_{0}$. The assumption 2 ) includes the requirement that the prefactor $\left(3 H^{2} / 5 \dot{\phi}\right)^{2}$ rapidly converges to some constant inside the bubble. If it changes by a large amount, the tilt of the spectrum may occur. $\tilde{C}_{\ell}^{(S)}$ is plotted in Fig. 2 . The detailed comparison of the resulting spectrum of the temperature fluctuation due to the scalar perturbation with the observation was discussed in Dr. Lineweaver's talk in this session. Therefore here we do not discuss it any further. 




FIG. 3. The normalized CMB anisotropy due to the wall fluctuation mode. The lines show, from top to bottom (at larger $l$ ), the cases of the universe with $\Omega_{0}=0.1,0.2,0.3,0.4,0.5$ and 0.6 .

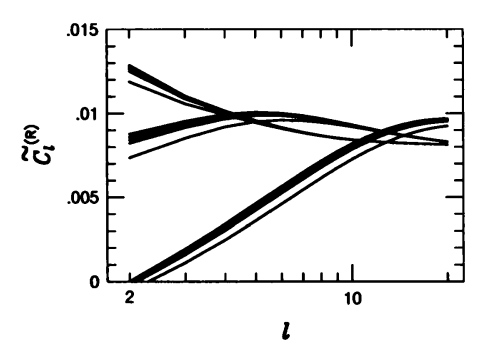

FIG. 4. The residual tensor CMB anisotropy spectra. The top (second and third) bunch of lines at $l=2$ are the universe with $\Omega_{0}=0.5$ (0.3 and 0.4$)$. Each bunch contains lines for several different models.

Next, let us consider the temperature fluctuation due to the tensor perturbation. The spectrum of the tensor perturbation was first obtained in Ref.(Tanaka et. al.(1997)) and it was proved that there is no supercurvature mode in the tensor perturbation (Tanaka et. al.(1997), Garriga et. al.(1997)). As in the scalar case, the shape of the continous spectrum of the tensor perturbation is almost model-independent except for the curvature scale modes with $p^{2} \lesssim 1$. However, different from the scalar case, the contribution of the curvature scale tensor perturbation to the temperature fluctuation can be very large. As mentioned above, this curvature scale perturbation is to be identified with the wall fluctuation mode. Since the subtle difference in the eigen value does not cause any significant change in the resulting temperature fluctuation, the contribution of the wall fluctuation modes to the temperature fluctuation is totally controled by its overall amplitude. Hence, we find that the resulting temperature fluctuation can be decomposed into two parts as $\ell(\ell+1) C_{\ell}^{(T)}=32 \pi G H^{2}\left(\mathcal{A} \tilde{C}_{\ell}^{(W)}+\tilde{C}_{\ell}^{(R)}\right)$. $\tilde{C}_{\ell}^{(W)}$ is model independently defined and is displayed in Fig. 3. $\mathcal{A}$ is defined by an appropreate integral of the spectrum of the tensor perturbation and heavily depends on the potential model. Then $\tilde{C}_{\ell}^{(R)}$, which is refered to as the gravitational wave mode, is separated as the remaining part of $C_{\ell}^{(T)}$. and is found to be almost model-independent as shown in Fig. 4.

If we assume the chaotic-type inflaton potential for the second inflation, the contribution of the gravitational wave mode is always smaller than that of the scalar mode. Thus, here we neglect the gravitational wave mode. On the other hand, the wall fluctuation mode can dominate $C_{\ell}$ if the amplitude $\mathcal{A}$ is large. $\tilde{C}_{\ell}^{(W)}$ has a large amplitude for the large angle fluctuation. Hence, if this mode dominates, the predicted spectrum of the temperature fluctuation contradicts with the observed one. Therefore, in order to construct a viable model, it must be required that the wall fluctuation mode does not dominate $C_{\ell}$. This condition gives a constraint on the model-dependent factor $\mathcal{A}$. Roughly speaking, the constraint on $\mathcal{A}$ can be 
interprited as a constraint on the model parameter: $\alpha:=\Delta V / 6 \pi G S_{1} \lesssim 70$, for typical values of $\Omega_{0}$ such as 0.3 or 0.4 . Here $S_{1}$ is something like the wall tension and $\Delta V$ is the difference between the heights of inflaton potential inside and outside the bubble wall.

It would be important to note that $\alpha>1$ is required for the existence of an appropriate Euclidean bounce solution. Even though there exists a bounce solution for any value of $\alpha$, the solution does not connects the state before and after the quantum tunneling for $\alpha<1$. The dissapearance of an appropriate bounce solution does not directly indicate that the tunneling process is suppressed in such a model. But we do not have any rigorous way to discuss the tunneling process in such a situation. The study in this direction is a curent topic to be developed. Relating to it, we proposed an alternative scenario to create an open universe (Sasaki et. al.(1997)), in which we do not consider the phase of the first inflation. Instead, we consider that the universe is created from nothing with bubble wall. In this case, we do not have to care for the constraint $\alpha>1$.

\section{Summary}

We have shown that in the standard inflation it is difficult to explain an open universe. However, this does not mean that the $\Lambda$-model is theoretically prefered to the open model. To show this, we explained the one-bubble inflation scenario as a solution to the difficulties in constructing an open universe in the context of the inflationary universe scenario. In the onebubble inflation scenario, one can calculate the predicted spectrum of the cosmological perturbation. The predicted spectrum is found to be rather model-independent except for the overall amplitude of the wall fluctuation mode, which is the curvature scale part of the tensor perturbation. By comparing the predicted spectrum with the observation, we obtain constraints on the potential model. A large region of the potential model parameters is still allowed to construct a viable model of the one-bubble inflation model. To conclude, the models that explain the creation of an open universe exist and so the open model is not less attractive than the $\Lambda$-model.

\section{References}

Garriga, J., Montes, X., Sasaki, M. and Tanaka, T. (1997) Canonical Quantization of Cosmological perturbation in the One Bubble Open Universe, astro-ph/9706229

Gott III, J.R., (1982) Nature, 295304

Sasaki, M., Tanaka, T. and Yakushige, T. (1997) Wall fluctuation modes and tensor CMB anisotropy in open inflation models, Phys. Rev., D 56, 616-624

Tanaka, T. and Sasaki, M. (1997) The Spectrum of Gravitational Wave Perturbations in the One-Bubble Open Inflationary Universe, Prog. Theor. Phys., 97, 243-262

Yamamoto, K., Sasaki, M. and Tanaka, T. (1996) Quantum fluctuations and CMB anisotropies in one-bubble open inflation models, Phys. Rev.,D 54, 5031-5048 